Supporting Information: Donor-Acceptor-Donor Type Liquid Crystal with a Pyridazine Core; Park, Kim, Lee, and Moon, July 12, 2006.

\title{
Donor-Acceptor-Donor Type Liquid Crystal with a Pyridazine Core
}

\author{
Yeon Sil Park, Dohyung Kim, Hoosung Lee, and Bongjin Moon* \\ Department of Chemistry, Sogang University, Shinsoo-dong 1, Mapo-gu, Seoul, Korea, 121-742, Korea
}

\section{Supporting Information}

All commercially obtained solvents and reagents were used without further purification except as noted below. Tetrahydrofuran (THF) was distilled from sodium benzophenone ketyl. $N, N$-dimethylformamide (DMF) was obtained from Aldrich Chemical Company, Inc. as anhydrous, $99+\%$ grade. Analytical thin-layer chromatography (TLC) was carried out on Merck silica gel $60 \mathrm{~F}_{254}$ glass plate and column chromatography was performed on Merck silica gel 60 (70 - 230 mesh).

${ }^{1} \mathrm{H}-\mathrm{NMR}$ and ${ }^{13} \mathrm{C}-\mathrm{NMR}$ spectra were obtained using a Varian Gemini-300 (300 $\mathrm{MHz}$ for ${ }^{1} \mathrm{H}$, and $75 \mathrm{MHz}$ for ${ }^{13} \mathrm{C}$ ), or a Varian Inova-500 $\left(500 \mathrm{MHz}\right.$ for ${ }^{1} \mathrm{H}$, and 125 $\mathrm{MHz}$ for ${ }^{13} \mathrm{C}$ ) spectrometer. Chemical shifts are reported relative to tetramethylsilane peak $(\delta 0.00)$ or solvent peak $\left(\delta 7.27\right.$ for $\mathrm{CDCl}_{3}$ in ${ }^{1} \mathrm{H} \mathrm{NMR}, \delta 77.2$ for $\mathrm{CDCl}_{3}$ in ${ }^{13} \mathrm{C}$ NMR). IR spectra were obtained using a Thermo-Nicholet Avartar-330 IR spectrometer with a single-bounce ATR (Ge crystal) accessory (Smart MIRacle). High resolution mass spectra were recorded on a 4.7 Tesla IonSpec ESI-FTMS. GC-MS were performed by the Organic Chemistry Research Center at Sogang University using an Agilent 5973N MSD spectrometer with an electron impact (EI) mode.

High resolution mass spectrum was recorded on a Micromass LCT ESI-TOF mass spectrometer.

The relative fluorescence quantum yield of $\mathbf{1}$ was acquired by using quinine sulfate as a standard. UV-visible absorbance spectra were obtained by an Agilent 8453 UV-visible spectrometer. Photoluminescence spectra were obtained by a Photon Technology International Spectrofluorophotometer (arc lamp supply: LPS-220, motor driver: MD-5020, PMT housing: 01-710). 
Supporting Information: Donor-Acceptor-Donor Type Liquid Crystal with a Pyridazine Core; Park, Kim, Lee, and Moon, July 12, 2006.

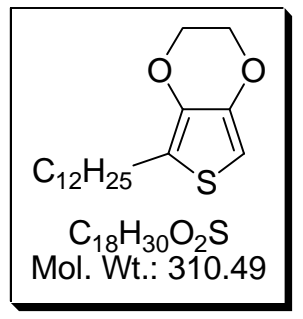

2-Dodecyl-3,4-ethylenedioxythiophene (3): A solution of 3,4ethylenedioxythiophene $(4.0 \mathrm{~g}, 28 \mathrm{mmol})$ in dry THF $(10 \mathrm{~mL})$ was added to a solution of $n$-BuLi (2.5 M in hexanes, $7.6 \mathrm{~mL}, 30.8 \mathrm{mmol}$ ) at $-78{ }^{\circ} \mathrm{C}$ under nitrogen atmosphere. The resulting solution was allowed to stir at $-78{ }^{\circ} \mathrm{C}$ for $2 \mathrm{~h}$, and dodecyl bromide (7.6 $\mathrm{mL}, 30.8 \mathrm{mmol}$ ) was added. The resulting orange solution was allowed to warm to room temperature and stirred overnight. The reaction was quenched by adding saturated $\mathrm{NH}_{4} \mathrm{Cl}(1 \mathrm{~mL})$. The solvent was evaporated and the residue was dissolved in $\mathrm{CH}_{2} \mathrm{Cl}_{2}$. The organic layer was washed with brine, dried over $\mathrm{MgSO}_{4}$, filtered, and evaporated. Purification of the crude product by flash column chromatography on silica gel (Hexane: $\left.\mathrm{CH}_{2} \mathrm{Cl}_{2}=2: 1\right)$ provided 3 as a white solid $(3.7 \mathrm{~g}, 42 \%):{ }^{1} \mathrm{H} \mathrm{NMR}\left(\mathrm{CDCl}_{3}, 300\right.$ MHz) $\delta 6.10$ (s, 1H), 4.16 (br s, 4H), 2.62 (t, $J=7.5 \mathrm{~Hz}, 2 \mathrm{H}), 1.58(\mathrm{~m}, 2 \mathrm{H}), 1.30$ - 1.22 (br m, $18 \mathrm{H}), 0.88(\mathrm{t}, J=6.8 \mathrm{~Hz}, 3 \mathrm{H}) ;{ }^{13} \mathrm{C} \mathrm{NMR}\left(\mathrm{CDCl}_{3}, 125 \mathrm{MHz}\right) \delta 141.6,137.5$, 118.7, 95.2, 64.9, 64.7, 32.1, 30.7, 29.9, $29.83(\times 2), 29.77,29.54,29.52,29.4,26.1,22.9$, 14.3; GC-MS (relative intensity) m/z $310\left(17.5 \%, \mathrm{M}^{+}\right), 311$ (3.6\%), $312(1.2 \%), 169$ (24.7\%, M-EDOT), 170 (2.7\%), 155 (100\%, M-EDOT-CH $\left.{ }_{2}\right), 156$ (12.0\%).

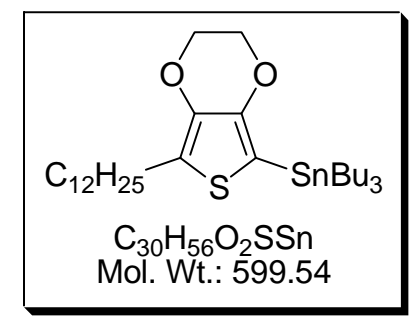

2-Dodecyl-5-tributylstannyl-3,4-ethylenedioxythiophene (4): A solution of $7(1.2 \mathrm{~g}, 3.8 \mathrm{mmol})$ in dry THF $(5 \mathrm{~mL})$ was added to a solution of $n$-BuLi $(2.5 \mathrm{M}$ in hexanes, $1.52 \mathrm{~mL}, 3.8 \mathrm{mmol}$ ) at $-78{ }^{\circ} \mathrm{C}$ under nitrogen atmosphere. The resulting solution was allowed to stir at $-78{ }^{\circ} \mathrm{C}$ for $2 \mathrm{~h}$, and tributylstannyl chloride $(1 \mathrm{~mL}, 3.8$ mmol) was added. The resulting yellow solution was allowed to warm to room temperature and stirred overnight. The reaction was quenched by adding saturated $\mathrm{NH}_{4} \mathrm{Cl}(1 \mathrm{~mL})$. The solvent was evaporated and the residue was dissolved in $\mathrm{CH}_{2} \mathrm{Cl}_{2}$. 
Supporting Information: Donor-Acceptor-Donor Type Liquid Crystal with a Pyridazine Core; Park, Kim, Lee, and Moon, July 12, 2006.

The organic layer was washed with brine, dried over $\mathrm{MgSO}_{4}$, and filtered. Concentration of the filtrate in vacuo afforded the crude product 4 as a yellow oil $(2.2 \mathrm{~g}$, $95 \%)$ which was used without further purification for the next step: ${ }^{1} \mathrm{H} \mathrm{NMR}\left(\mathrm{CDCl}_{3}\right.$, $300 \mathrm{MHz}) \delta 4.13(\mathrm{~m}, 4 \mathrm{H}), 2.62(\mathrm{t}, J=7.5 \mathrm{~Hz}, 2 \mathrm{H}), 1.61-1.51$ (br m, $8 \mathrm{H}), 1.37-1.25$ (br m, $24 \mathrm{H}), 1.07$ (t, $J=8.1 \mathrm{~Hz}, 6 \mathrm{H}), 0.89(\mathrm{~m}, 12 \mathrm{H})$.

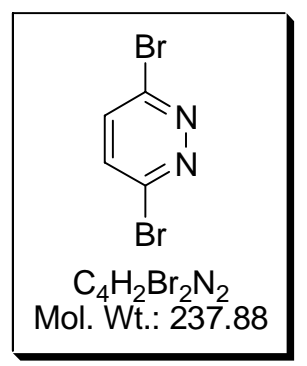

3,6-Dibromopyridazine (5) ${ }^{1}$ : A mixture of maleic hydrazide (1.1 g, 10 mmole) and $\mathrm{PBr}_{5}(4.7 \mathrm{~g}, 11 \mathrm{mmol})$ was heated at $100{ }^{\circ} \mathrm{C}$ until evolution of white fumes of hydrogen bromide ceased $(\sim 2 \mathrm{~h})$. After cooling, the orange residue was poured into ice water and the resulting mixture was extracted with $\mathrm{CH}_{2} \mathrm{Cl}_{2}(3 \times 20 \mathrm{~mL})$. The organic layer was washed with saturated aqueous $\mathrm{NaHCO}_{3}$ solution, dried over $\mathrm{MgSO}_{4}$, filtered, and concentrated. Purification of the crude product by flash column chromatography on silica gel $\left(\mathrm{CH}_{2} \mathrm{Cl}_{2}\right)$ provided 5 as a white solid $(1.20 \mathrm{~g}, 50 \%)$ : ${ }^{1} \mathrm{H} \mathrm{NMR}\left(\mathrm{CDCl}_{3}, 300\right.$ $\mathrm{MHz}) \delta 7.53$ (s, 2H); ${ }^{13} \mathrm{C} \mathrm{NMR}\left(\mathrm{CDCl}_{3}, 75 \mathrm{MHz}\right) \delta 147.8$, 133.5; GC-MS (relative intensity) m/z 241 (2.5\%), 240 (49\%, M+4), 239 (5.3\%), 238 (100\%, M+2), 236 (51.9\%, $\left.\mathrm{M}^{+}\right), 212$ (15.5\%, M-N $\left.2+4\right), 210$ (32.1\%, M-N $\left.2+2\right), 208\left(16.4 \%, \mathrm{M}_{2}\right), 131$ (79.9\%, $\left.\mathrm{M}-\mathrm{N}_{2}-\mathrm{Br}+2\right), 129$ (82.3\%, M-N $\left.-\mathrm{Br}\right), 51$ (48.7\%, $\left.\mathrm{M}_{2} \mathrm{~N}_{2}-\mathrm{Br}_{2}+1\right), 50$ (71.8\%, $\mathrm{M}_{-} \mathrm{N}_{2}-\mathrm{Br}_{2}$ ).

${ }^{1}$ Komin, A. P.; Carmack, M. J. Heterocyclic Chem. 1976, 13, 13. 
Supporting Information: Donor-Acceptor-Donor Type Liquid Crystal with a Pyridazine Core; Park, Kim, Lee, and Moon, July 12, 2006.

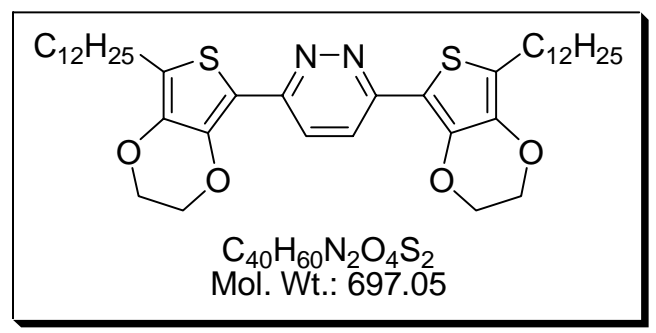

\section{3,6-Bis(5-(2-dodecyl-3,4-ethylenedioxythienyl))pyridazine $(1)^{2}$ :}

3,6-Dibromopyridazine (5) (214 mg, $0.9 \mathrm{mmol})$, tributylstannyl compound 4 (1.6 g, 2.7 $\mathrm{mmol})$, and $\mathrm{PdCl}_{2}\left(\mathrm{PPh}_{3}\right)_{2}(126 \mathrm{mg}, 0.18 \mathrm{mmol})$ were mixed in anhydrous DMF $(7 \mathrm{~mL})$ and heated at $80{ }^{\circ} \mathrm{C}$ for $16 \mathrm{~h}$ under nitrogen atmosphere. After cooling the reaction mixture, the solvent was removed by distillation under reduce pressure, and the residue was dissolved in $\mathrm{CH}_{2} \mathrm{Cl}_{2}(30 \mathrm{~mL})$. Saturated $\mathrm{KF}$ solution $(20 \mathrm{~mL})$ was added and this mixture was stirred for $1 \mathrm{~h}$. The organic layer was separated, and washed with saturated aqueous $\mathrm{NaHCO}_{3}$ solution, dried over $\mathrm{Na}_{2} \mathrm{SO}_{4}$, filtered, and concentrated. Purification of the crude product by flash column chromatography on silica gel (Hexane: $\mathrm{CH}_{2} \mathrm{Cl}_{2}=$ 1:4) with a pad of $\mathrm{KF}$ provided 1 as a pale yellow solid (446 $\mathrm{mg}, 71 \%$ ): ${ }^{1} \mathrm{H} \mathrm{NMR}$ $\left(\mathrm{CDCl}_{3}, 300 \mathrm{MHz}\right) \delta 7.94(\mathrm{~s}, 2 \mathrm{H}), 4.33(\mathrm{~m}, 4 \mathrm{H}), 4.24(\mathrm{~m}, 4 \mathrm{H}), 2.67(\mathrm{t}, J=7.5 \mathrm{~Hz}, 4 \mathrm{H})$, 1.63 (m, 4H), $1.34-1.18$ (br m, $36 \mathrm{H}), 0.88$ (t, $J=6.8 \mathrm{~Hz}, 6 \mathrm{H}) ;{ }^{13} \mathrm{C} \mathrm{NMR}\left(\mathrm{CDCl}_{3}, 125\right.$ MHz) $\delta 141.6,137.5,118.7,95.2$, 64.9, 64.7, 32.1, 30.7, 29.9, $29.83(\times 2), 29.77,29.54$, 29.52, 29.4, 26.1, 22.9, 14.3; IR (KBr) 3101, 2980, 2930, 2880, 1536, 1499, 1440, 1177 , 1065, 930, 906, 846, $734 \mathrm{~cm}^{-1}$; HRMS (ESI) m/z 697.4074 (697.4073 calculated for $\left.\mathrm{C}_{40} \mathrm{H}_{61} \mathrm{~N}_{2} \mathrm{O}_{4} \mathrm{~S}_{2}\left[\mathrm{M}+\mathrm{H}^{+}\right]\right)$.

${ }^{2}$ Stille, J. K. Angew. Chem., Int. Ed. Engl., 1986, 25, 508. 
$n$

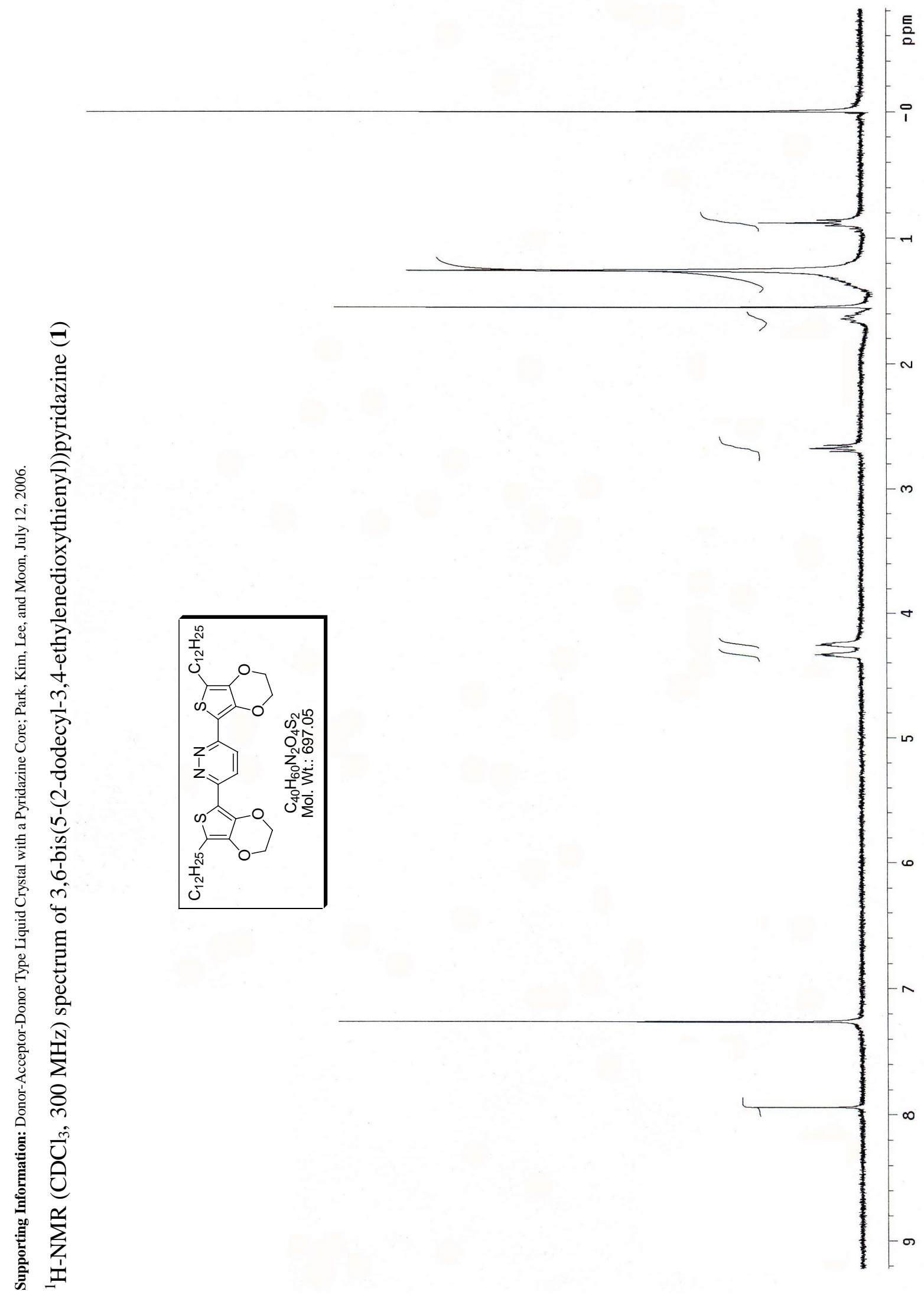




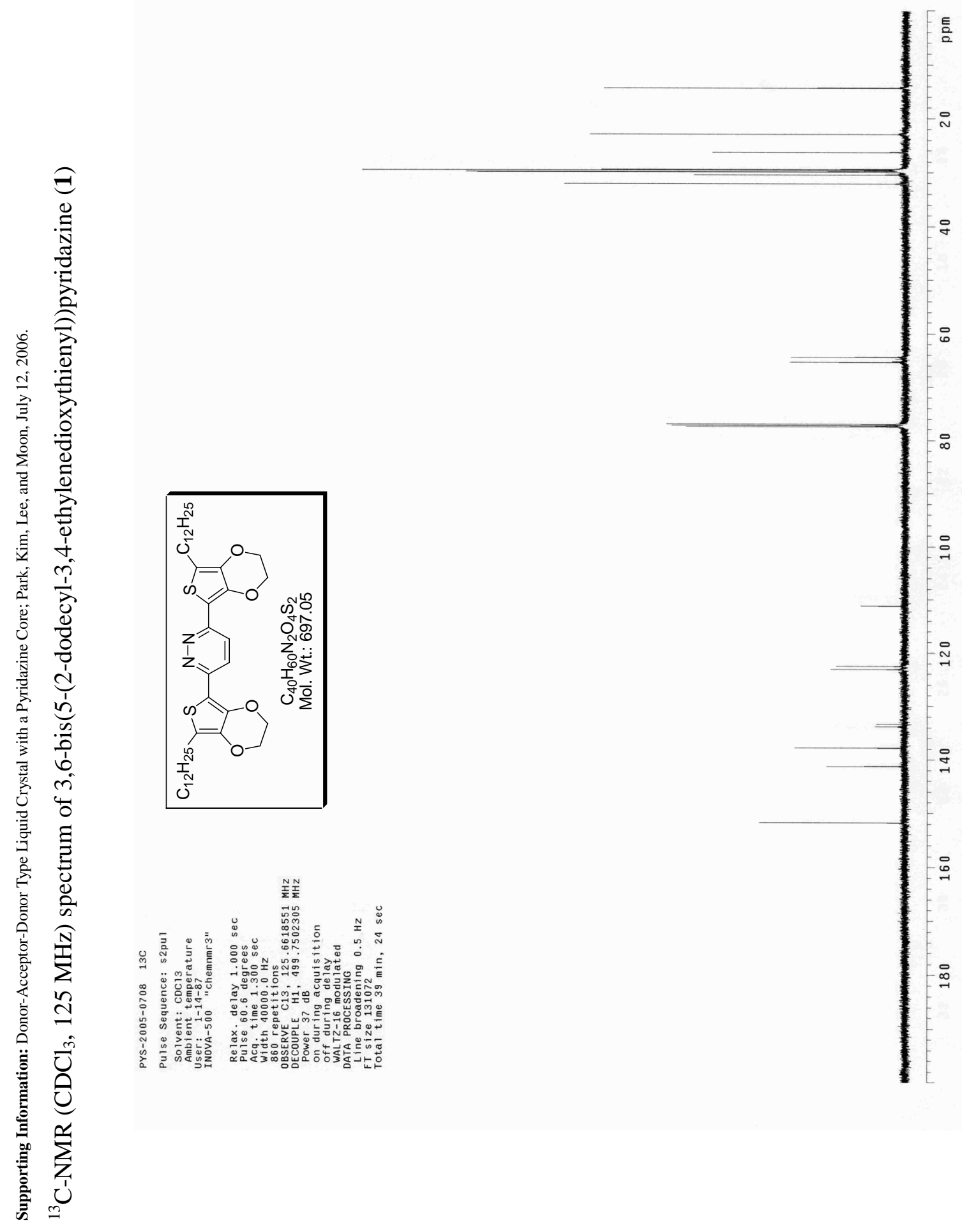


Supporting Information: Donor-Acceptor-Donor Type Liquid Crystal with a Pyridazine Core; Park, Kim, Lee, and Moon, July 12, 2006.

HRMS of 3,6-bis(5-(2-dodecyl-3,4-ethylenedioxythienyl))pyridazine (1)

pys \#1

pys001 $93(0.930) \mathrm{Cm}(33: 94)$

100
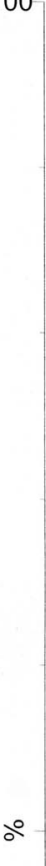

$\therefore$
KC434 Install AK

697.4074

$\mathrm{C}_{12} \mathrm{H}_{25} \sim \mathrm{S} \quad \mathrm{N}-\mathrm{N} \quad \mathrm{S}-\mathrm{C}_{12} \mathrm{H}_{25}$<smiles>Oc1sc(-c2cccnn2)c2c1OCCO2</smiles>

$\mathrm{C}_{40} \mathrm{H}_{60} \mathrm{~N}_{2} \mathrm{O}_{4} \mathrm{~S}_{2}$

Exact Mass: 696.3994

Mol. Wt.: 697.0454

m/e: 696.3994 (100.0\%), 697.4028 (44.5\%),

$698.4062(9.6 \%), 698.3952(8.9 \%)$,

699.3986 (3.9\%), 697.3988 (1.6\%), $699.4095(1.4 \%)$

04-Aug-2005

15:35:40

TOF MS ES+

$1.30 \mathrm{e} 3$ 


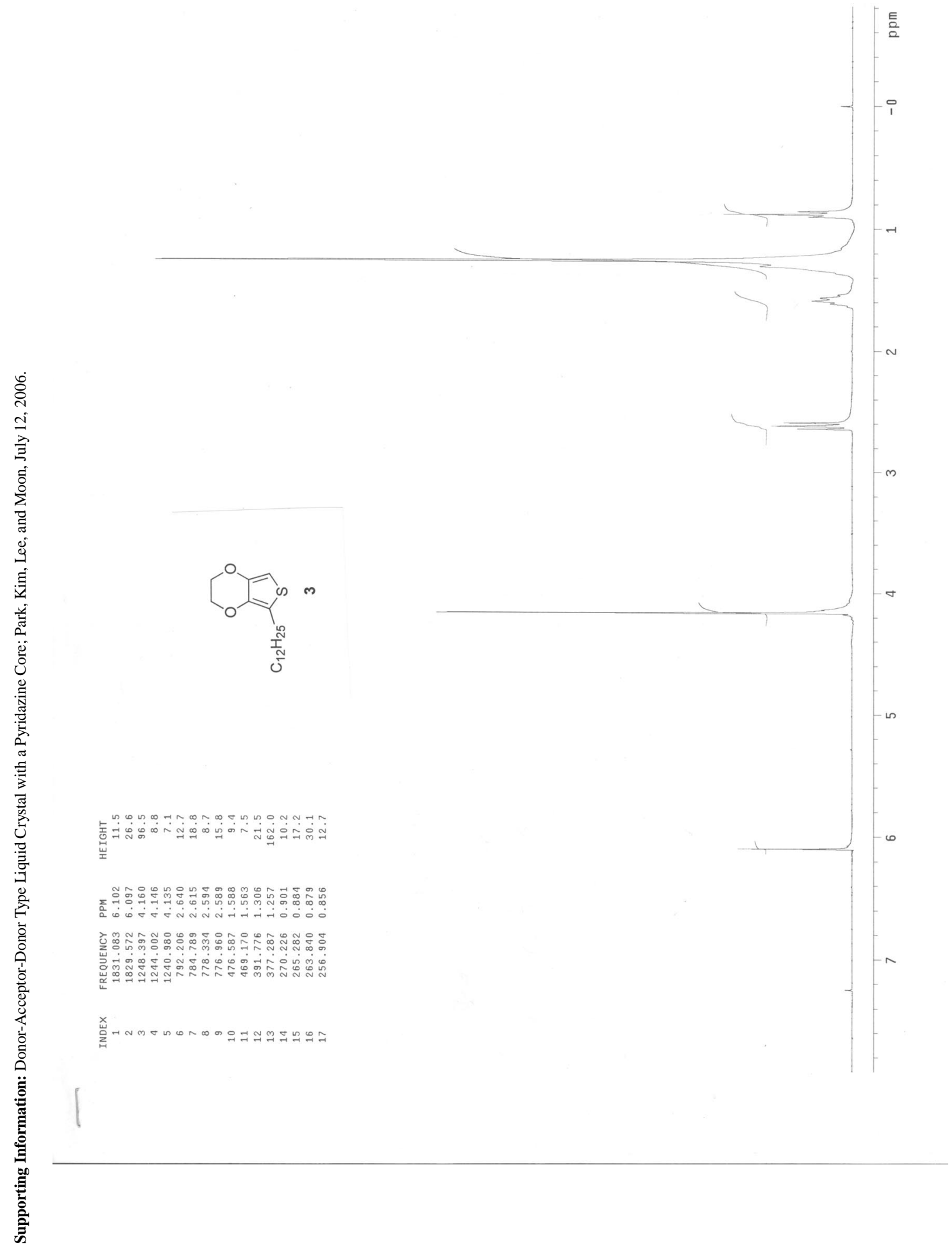




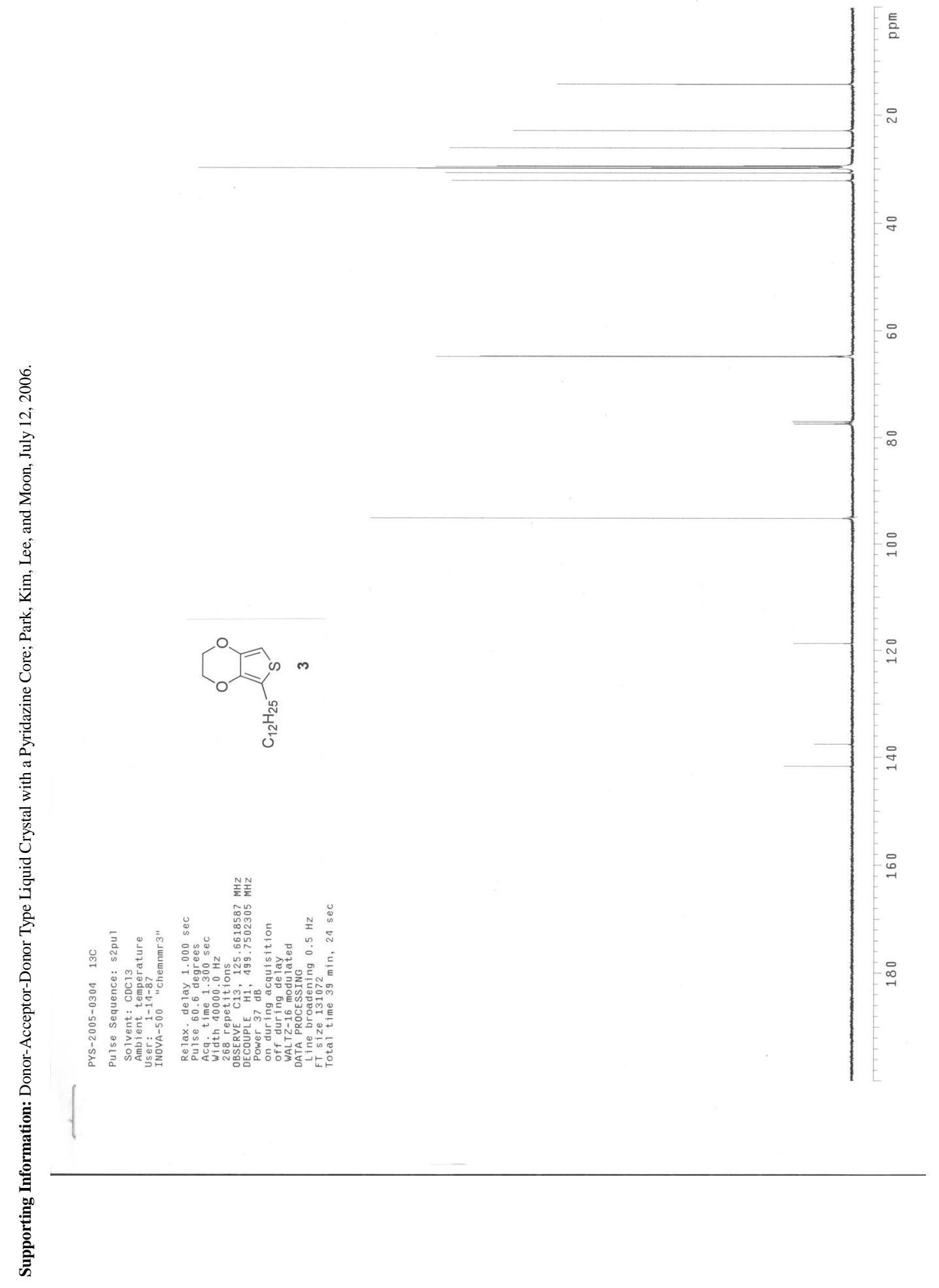


in

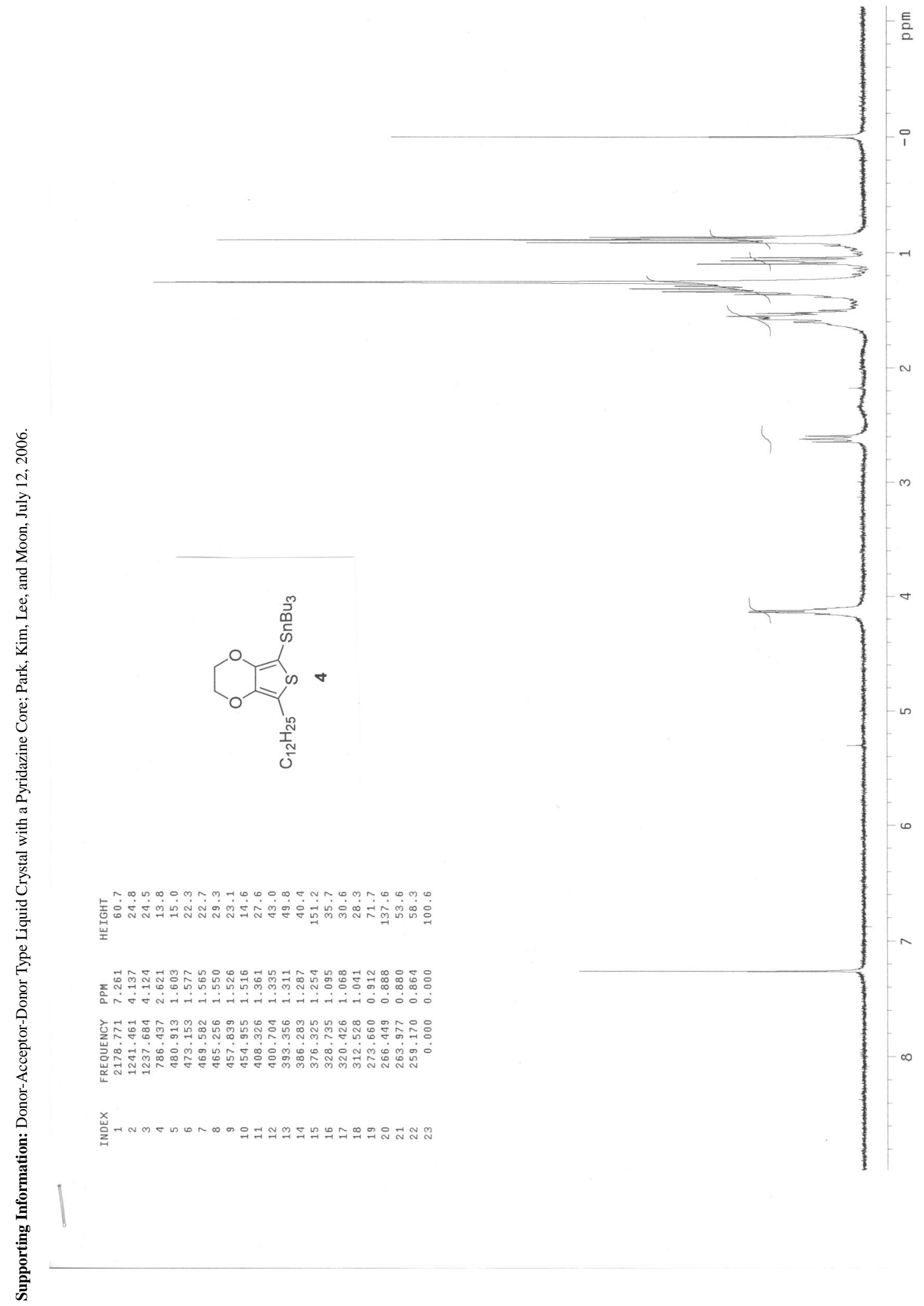


$\overline{5}$

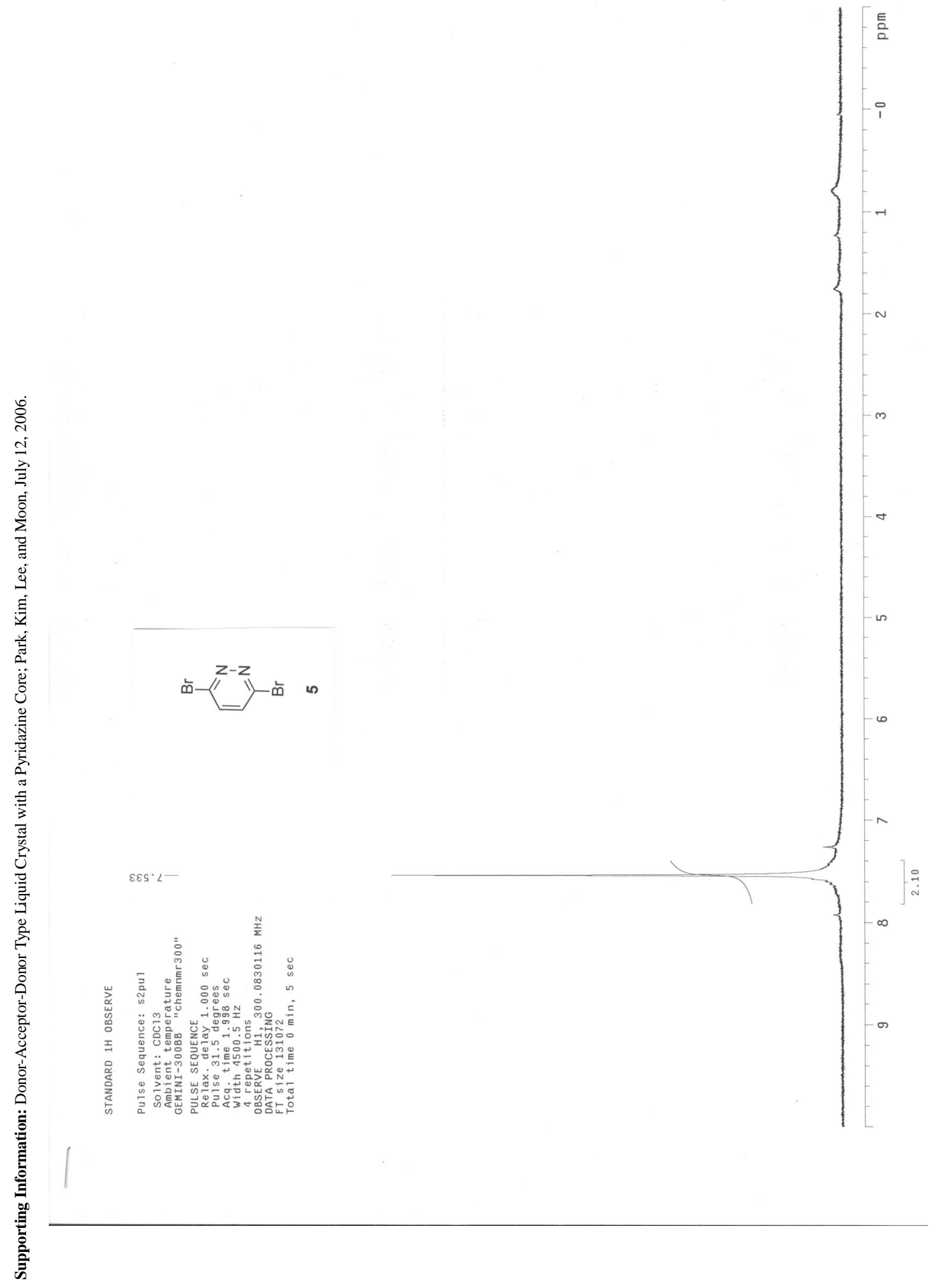


$\frac{\pi}{n}$

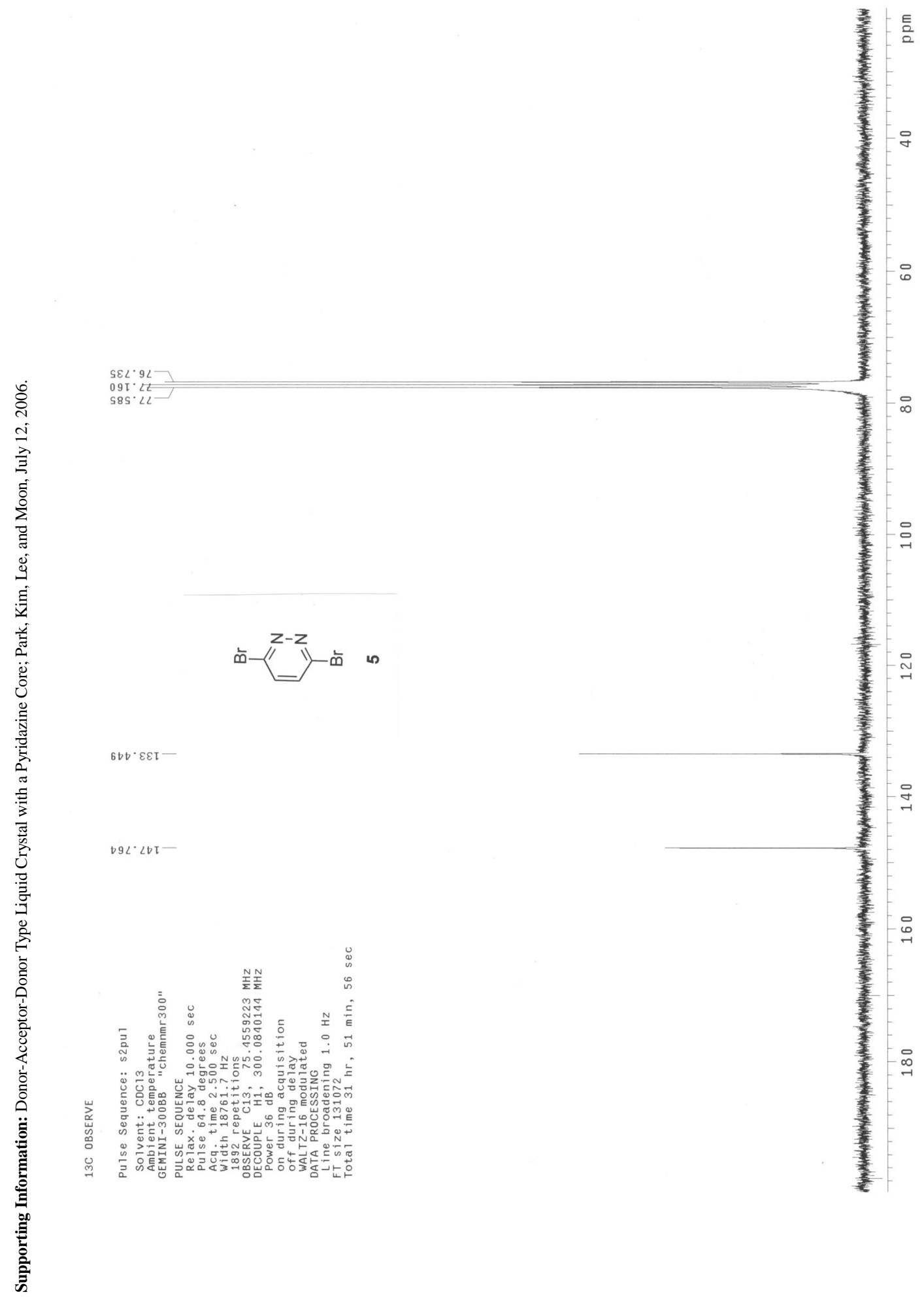

